\title{
TRAVELING WAVE STRUCTURE OPTIMIZATION FOR THE NLC*
}

\author{
Zenghai Li, Karl L. Bane, Roger H. Miller, Tor O. Raubenheimer, Juwen Wang, Ronald D. Ruth, \\ $S L A C$, USA
}

\begin{abstract}
The JLC/NLC linac must accelerate multi-bunch beams in order to obtain a luminosity $>3 \times 10^{34} \mathrm{~cm}^{-2} \mathrm{sec}^{-1}$ at a center of mass energy of $1 \mathrm{TeV}$. It is essential for the structure design to minimize the long and short-range dipole wakefields to prevent emittance degradation and the beam breakup instability (BBU). In addition, the structures must operate at a high RF gradient to minimize the linac cost. High-power testing of prototype structures at SLAC has shown noticeable damage. The damage is largest in the front of the structure, where the group velocity is high, and there is minimal or no damage at the back end, where the group velocity is low. Theoretical analysis using a simple circuit model suggests using structures with a lower group velocity, on the order of a few percent, would be a way of avoiding damage. For the standard $2 \pi / 3$ accelerating mode, it is difficult to lower the group velocity without losing efficiency or increasing the wakefields. With this in mind, we have taken the phase advance as an additional parameter in structure optimization. We found that a low group velocity structure at higher phase advance can maintain high RF efficiency and low wakefields. In this paper, we study the impact of phase advance on structure performance. We then optimize the NLC S-band and X-band structures to meet design requirements.
\end{abstract}

\section{INTRODUCTION}

The NLC[1] is an $\mathrm{e}^{+} \mathrm{e}^{-}$collider designed to provide a luminosity of $3.4 \times 10^{34} \mathrm{~cm}^{-2} \mathrm{sec}^{-1}$ at a center-of-mass energy of $1 \mathrm{TeV}$. The linacs of such a project must be able to accelerate high-current multi-bunch beams efficiently. The beam consists of a 265 ns bunch train with an average current of $0.9 \mathrm{~A}$ and a 1.4-ns bunch spacing. The emittance degradation and breakup instability of the beams in the linac depend on both the wakefields and the alignment of the RF structures. To achieve a realistic alignment tolerance for the structures, we need to design the structures with low dipole wakefields. The NLC consists of three types linacs, operating at L-band (1.428$\mathrm{GHz})$, S-band (2.856-GHz) and X-band (11.424-GHz) frequencies. While less important at L-band, minimizing the dipole wakefields in both the S-band and X-band structures is a major design issue. The stringent requirement on the dipole wakefields has a direct impact on the choice of basic structure parameters. The high gradient requirement for the $\mathrm{X}$-band structures puts additional constraints on the design. Tradeoffs need to be made between RF efficiency, wakefields and breakdown

\footnotetext{
* Work supported by the U.S. DOE Contract No. DE-AC03-76SF00515.
}

limitations. In this paper, we will try to address some of the structure design issues. We will use the phase advance as an additional parameter in structure optimization to meet the design requirements.

\section{DESIGN CONSIDERATIONS}

\section{Structure aperture: $a$}

The aperture of the structure is constrained by singlebunch wakefields, which scale as $a^{-3.8}$. BNS damping can effectively suppress the associated head-tail effects by accelerating the beam off-crest to induce a correlated energy spread across the bunch. The rf phases for BNS damping and the residual energy spread become larger as the iris aperture becomes smaller. The requirements on emittance and RF efficiency set a lower bound on the structure aperture. It is shown in Ref. 2 that using aperture smaller than $0.18 \lambda$ in the $\mathrm{X}$-band linac will result in emittance dilution and loss in rf efficiency.

\section{Dipole mode detuning}

Coherent cancellation among the dipole modes is realized by detuning the modes in a prescribed manner to suppress the long-range dipole wakefields. In addition to the detuning, there are specific requirements on the dispersion relations of the dipole modes in order to obtain a smooth spectrum such that effective wakefield suppression will extend over a longer distance. To achieve this, the bandwidth of the dipole modes at the zero phase advance is required to overlap the bandwidth at the $\pi$ phase advance. In this way, the modes that touch the ends of the structure will not be synchronous with the beam and therefore will have low kick factors. In another words, the slopes of the dispersion curves are required to change sign along the structure. Figure 1 shows one example that satisfies this criterion. Dispersion curves for five representative cells along the structure are shown (notice the change in slopes). The modes at the two ends are far from the synchronous phase.

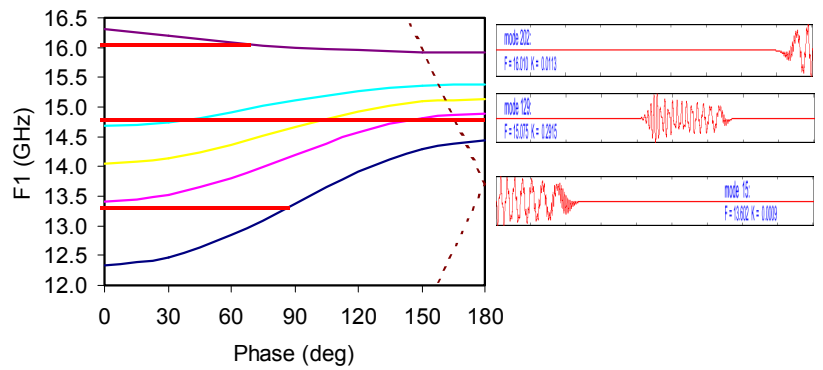

Figure 1 Dipole dispersion and typical modes in a traveling wave structure. 


\section{Group velocity}

The structures need to operate at high gradient reliably. High power tests on the X-band prototypes have shown noticeable breakdown damage [3]. Studies using a simple circuit model [4] suggested that the rf power needs to travel slowly though the structure to reduce this damage. The idea is to reduce the amount of energy that can go into an individual breakdown, minimizing the surface erosion. The present thought is to design the X-band structure with a lower group velocity of about $0.05 \mathrm{c}$ or $0.03 \mathrm{c}$, as compared to the $0.12 \mathrm{c}$ for the RDDS1 structure.

\section{Structure filling time $t_{l}$}

The optimal filling time of a traveling wave structure depends on the ratio of the beam loading and the RF acceleration voltages. In a constant gradient structure, the beam-loading gradient and the RF gradient are given by

$$
\begin{aligned}
& G_{b}=-\frac{I_{0} r}{2}\left(1-\frac{2 \tau e^{-2 \tau}}{1-e^{-2 \tau}}\right) \\
& G_{r f}=\sqrt{\frac{P_{i n} r}{L}\left(1-e^{-2 \tau}\right)}
\end{aligned}
$$

where $\tau=\omega t_{f} / 2 Q$ is the attenuation factor. For a given center of mass energy at the interaction point, the RF efficiency is proportional to $G_{L}^{2} / U_{r f}$ with $G_{L}=G_{r f} G_{b}$ the loaded gradient and $U_{r f}=P\left(t_{f}+t_{b}\right)$ the energy stored in the RF pulse. For the NLC RF power and beam loading parameters, the $\tau$ dependences of these functions are plotted in Fig. 2.
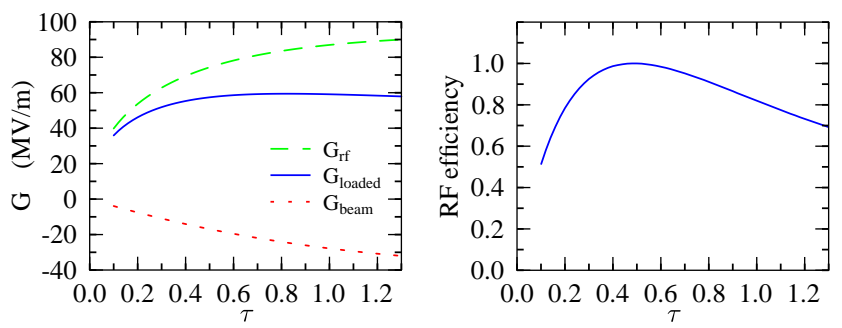

Figure 2 Gradient and efficiency as functions of $\tau$. The beam loading current is 0.9 -amp. The RF power per unit structure length is $97-\mathrm{MW} / \mathrm{m}$.

The optimal $\tau$ is in between 0.4 to 0.6 . The lower efficiency at a larger $\tau$ is due to large beam loading and more stored energy in the RF pulse. The X-band structure will be designed with a $\tau$ of about 0.5 , which corresponds to a filling time of $\sim 100 \mathrm{~ns}$.

\section{IMPACT OF PHASE ADVANCE}

With the standard $2 \pi / 3$ phase advance, it is difficult to design structures to meet all the constraints. For this reason, we have taken the phase advance of the acceleration mode as an additional parameter.

The impact of phase advance on some of the RF parameters (X-band) are shown in Fig. 3. The three curves correspond to three cells with different iris radii. The shunt impedance has a broad optimal between 120 to 150 degrees, with a slight dependence on iris opening.
The dipole modes are detuned by a similar amount at these phases. The group velocity of the fundamental mode, which is proportional to $\sin (\varphi)$ as expected, can be effectively lowered by designing the structure to have a high phase-advance. The group velocity of the dipole mode, which is not shown here, is also sensitive to the phase advance. This can be used as a knob to tune the dispersion properties of the dipole modes. The kick factors are lower in the high phase advance design which is a plus for minimizing the dipole wakefield.
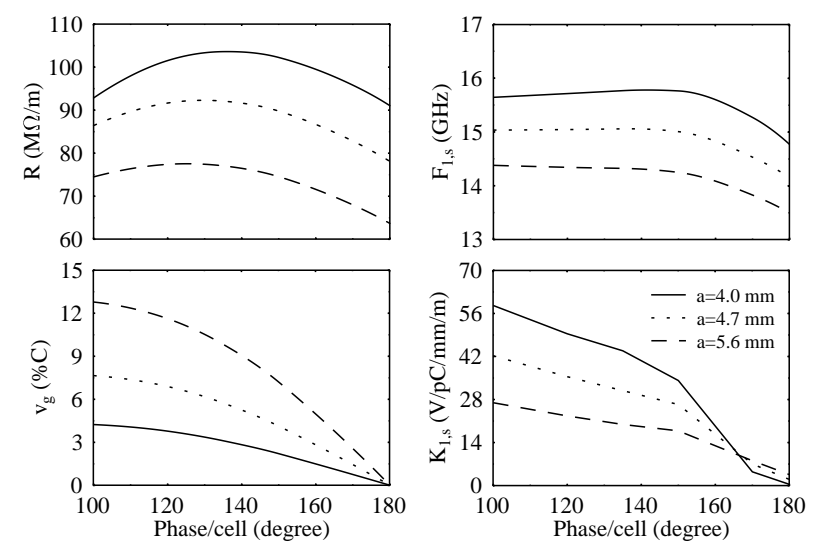

Figure 3 RF parameters v.s. phase advance for three aperture openings at X-band

\section{S-BAND STRUCTURE}

The $3 \pi / 4$ mode was chosen for the S-band pre-linac structure [5]. The choice of this phase advance is to resolve two design difficulties: 1) to generate proper dipole modes to suppress wakefields for both 1.4-ns and 2.8-ns bunch spacings; 2) to optimize the filling time for $\mathrm{RF}$ efficiency. Since the dipole frequency at S-band is four times lower than at X-band, significantly reducing the wake envelope after the bunch spacing of $1.4 \mathrm{~ns}(4 \mathrm{~S}-$ band wavelengths) by detuning alone is difficult. Another technique to minimize the wakefield, is to put the bunches at zero crossings of the wakefield; this requires a center frequency of 3.93-GHz for the dipole modes. This center frequency is about $2 \%$ lower than the optimized design with $2 \pi / 3$ phase advance. Shifting the frequency by opening up iris will lead to a 7\% loss in RF efficiency due to a shorter filling time. The dipole dispersion properties also become problematic. At $3 \pi / 4$ phase advance, we can effectively lower the dipole frequency while maintaining large apertures, a good filling time, and actually improving the rf efficiency. The dipole dispersion can also be designed to satisfy the requirements as mentioned in Sec.2; see details in Ref. [5].

\section{LOW V GX-BAND STRUCTURE}

The X-band structure needs to have a starting group velocity of $0.05 \mathrm{c}-0.03 \mathrm{c}(\mathrm{vg} 5, \mathrm{vg} 3)$ to cope with the breakdown damage problem. With the $2 \pi / 3$ mode, it is difficult to design a structure with an average iris aperture of $a / \lambda=0.18$ without loosing significant RF efficiency. 
This leads to the choice of higher phase advance, taking the advantages of low group velocity and high shunt impedance. One concern with higher phase advance is the narrower bandwidth since the synchronous phase now is closer to $\pi$-phase and this limits how high the phase advance can be. The $5 \pi / 6$ (150 degrees) is chosen for the present design. With this mode, the 0.05 c group velocity can be easily obtained for $a / \lambda=0.18$. For the $0.03 \mathrm{c}$ design, one needs a thicker disk, which reduces the shunt impedance slightly.

\section{Structure length}

To obtain an optimal filling time, the structure length should scale roughly proportional to the group velocity. Simple scaling from the RDDS1[6,7] which has a group velocity of $0.12 \mathrm{c}$, the length of $\mathrm{vg} 5$ and $\mathrm{vg} 3$ structures would be about $0.75-\mathrm{m}$ and $0.45-\mathrm{m}$ respectively. Since the ends of the structure dominate the cost, longer structures are desirable. A reasonable choice of structure length is thought to be 0.9 meters. In the vg5 structure, the $90 \mathrm{~cm}$ length can be reached by slightly extending the filling time. In the vg3 structure, we used a reversed taper on the disk thickness (from thick to thin) to maximize the average group velocity. On the other hand, with a fixed input power per unit length, the input power per structure is higher for a longer length. To minimize the breakdown damage (by reducing the power flow), it may be sensible to reduce the structure length. A length of $0.6-\mathrm{m}$ seems to be a good compromise between cost and reducing breakdown energy. However, high power tests are needed to verify these various possibilities.

One concern in the wakefield design with a shorter structure is the fewer modes in the dipole spectrum. This may weaken the cancellation due to the decoherence of the modes however, this issue will not be addressed here.

\section{Low $v_{g}$ structures}

Three types of low $\mathrm{v}_{\mathrm{g}}, 5 \pi / 6$ phase advance structures were studied. The main RF parameters of these structures are listed in Table 1 . The gradients were calculated with a nominal input power of $97-\mathrm{MW} / \mathrm{m}$ and loading current of 0.9 -amps. Both the vg3 and vg5 0.9-m structures can produce an unloaded gradient of $70-\mathrm{MV} / \mathrm{m}$. The vg3 0.6$\mathrm{m}$ structure has a lower unloaded gradient. The loaded gradient for these three designs are all above $50-\mathrm{MV} / \mathrm{m}$. Notice that the input power per structure for the $0.6-\mathrm{m}$ design is $30 \%$ less.

\section{Bandwidth, structure dispersive effects}

Dispersion is a concern in the low $\mathrm{v}_{\mathrm{g}}$ design because of the narrower bandwidth associated with the low $\mathrm{v}_{\mathrm{g}}$ and high phase advance. One possible consequence of this effect are distortions in the rf pulse which may induce a large energy variation across the bunch train. Figure 4 shows a snapshot of the accelerating field $E_{z}$ in the $\mathrm{vg} 5$ $0.9-\mathrm{m}$ structure at $\mathrm{t}=100 \mathrm{~ns}$. The smooth curve (dashed) is the RF pulse with the dispersive effect off. The solid curve is the RF pulse with the dispersive effect on. The acceleration is an integral of this field. Simulations show that the energy variation caused by the dispersive effect is not significant and is within tolerances. If necessary, additional pulse shaping could be used to compensate the energy variation.

Table 1 Parameters for three low $\mathrm{v}_{\mathrm{g}}$ structures.

\begin{tabular}{|l|c|c|c|}
\hline Parameters & Vg3_60 & Vg3_90 & Vg5_90 \\
\hline $\mathrm{L}_{\text {struct }}(\mathrm{m})$ & 0.601 & 0.908 & 0.908 \\
\hline $\mathrm{N}_{\text {cell }}$ & 55 & 83 & 83 \\
\hline $\mathrm{T}_{\mathrm{f}}(\mathrm{ns})$ & 110 & 120 & 121 \\
\hline$\tau$ & 0.529 & 0.515 & 0.508 \\
\hline $\mathrm{V}_{\mathrm{g}} / \mathrm{c}(\%)$ & $3.16-1.05$ & $3.20-2.10$ & $5.06-1.14$ \\
\hline $\mathrm{F}_{1, \mathrm{c}}(\mathrm{GHz}) / 0.1 / 4 \sigma$ & 15.20 & 15.27 & 15.18 \\
\hline Power in $(\mathrm{MW})$ & 58.85 & 87.5 & 87.5 \\
\hline $\mathrm{G}_{\mathrm{L}} / \mathrm{G}_{\mathrm{UNL}}(\mathrm{MV} / \mathrm{m})$ & $51.1 / 64.7$ & $53.5 / 69.3$ & $54.6 / 70.0$ \\
\hline
\end{tabular}

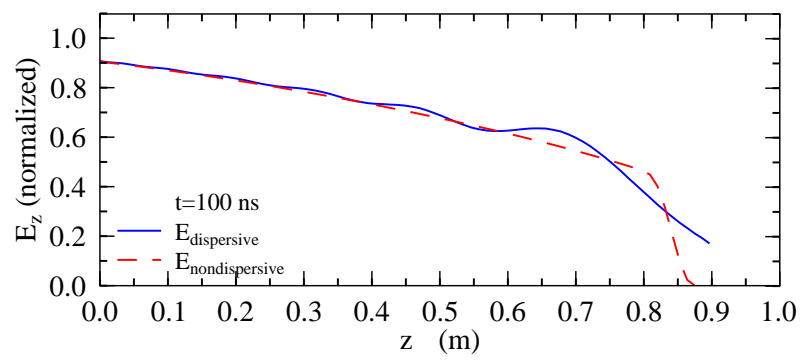

Figure 4 A snapshot Ez fields in the vg5 0.9-m structure: solid) dispersive on; dashed) dispersive off.

\section{SUMMARY}

In this paper, we have presented ideas of using phase advance as an additional parameter to optimize both the dipole and the fundamental modes, and showed that low group velocity structures can be designed with high RF efficient using a high phase advance mode.

The authors would like to acknowledge the structures and the NLC linac group meetings for helpful discussions and support.

\section{REFERENCES}

[1] ZDR report for the Next Linear Collider, LBNL-PUB5424, SLAC Report 474, UCRL-ID-124161, 1996.

[2] G. Stupakov and Z. Li, Varying a/ $\lambda$ in NLC Structures - BNS Damping and Emittance Growth, LCC-0063.

[3] C. Adolphsen, these proceedings.

[4] C. Adolphsen, NLCTA Structure Operation Results, Structure Breakdown Workshop, SLAC, August 2000.

[5] K. Bane and Z. Li, Dipole Mode Detuning in the Injector Linacs of the NLC, SLAC-AP-134, LCC0043, Dec. 2000

[6] Z. Li, et al, RDDS1 Cell Design and Optimization for the Linear Collider Linacs, proceedings of PAC1999, New York, 1999.

[7] J. Wang, et al, Design Fabrication and Measurement of the RDDS1 Structure, proceedings of LINAC2000, Monterey, CA, 2000 